\title{
SENTINEL-1 DATA TO MAP FLOODED AREAS: THE ROLE OF INSAR COHERENCE AND POLARIMETRIC INFORMATION
}

\author{
Papastergios A. ${ }^{1}$, Chini M. ${ }^{2}$ and Parcharidis . $^{1}$ \\ ${ }^{1}$ Harokopio University of Athens, Department of Geography, 17671, Athens, Greece, \\ gs21131@hua.gr,parchar@hua.gr \\ ${ }^{2}$ Luxembourg Institute of Science and Technology (LIST), 41 rue du Brill, Belvaux, 4422, \\ Luxembourg,marco.chini@list.lu
}

\begin{abstract}
SAR earth observation data can provide high quality flood maps and information to better assess the flood risk accordingly planning as well as to support civil protection authorities during emergency phase. The scope of this paper is to create flood extent maps from a series of SAR scenes of the Evros basin which represents a transboundary floodplain. The study uses time series SAR images of Sentnel-1 ESA's Copernicus satellite system covering the period October 2014 to May 2015.

The methodology tries to identify the flood that occurs in three main land cover classes, such as urban areas, bare or poorly vegetated soil and vegetated areas, taking advantage of co- and cross-polarized SAR backscattering channels, and the InSAR coherence to better characterize the landscape. Dual-pol SAR data provides the opportunity to have a better understanding and interpretation of flood detection due to way different land cover react to different polarizations. Thus, with the implementation of InSAR coherence estimation we may achieve a better record and knowledge of the flooded areas, over time, in the specific region.

Keywords: Flood mapping, SAR, Evros, Hydrology.
\end{abstract}

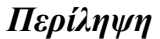

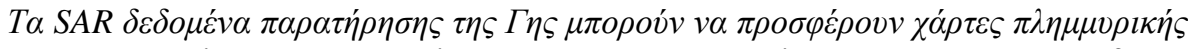

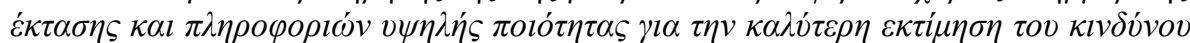

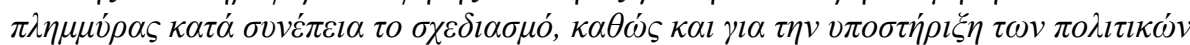

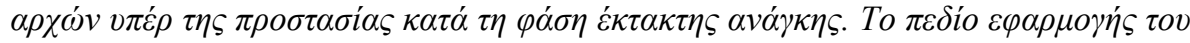

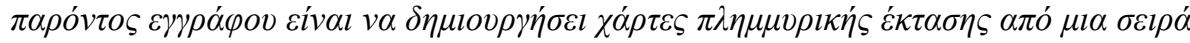

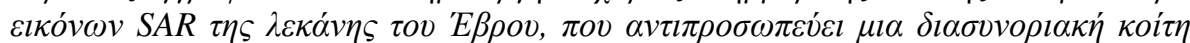

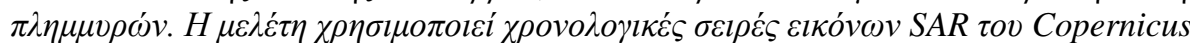

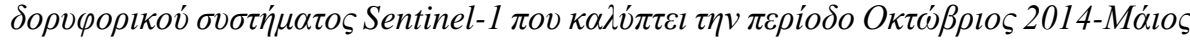
2015.

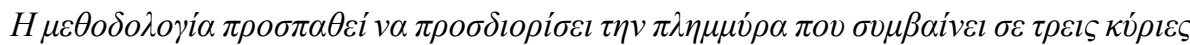

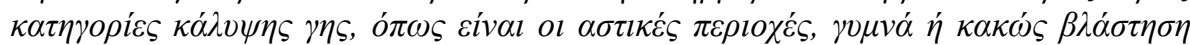

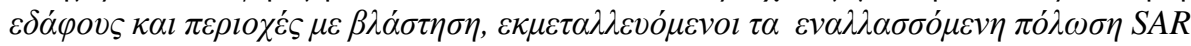

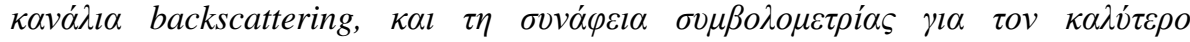

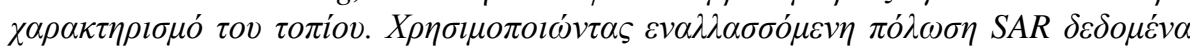

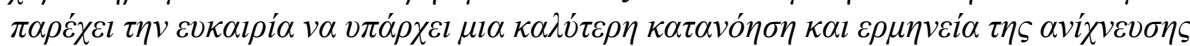

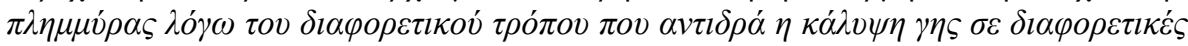




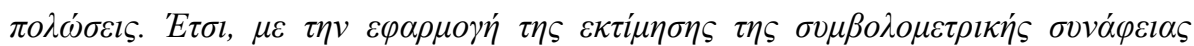

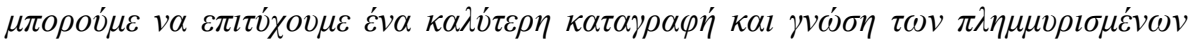

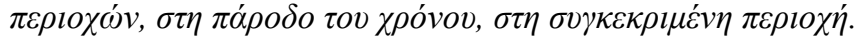

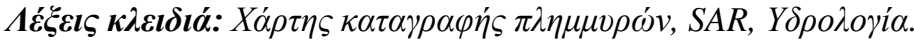

\section{Introduction}

Presently, the most common approach for using EO data for flood mapping is based on SAR images. SAR is characterized by a good sensitivity to water and is able to provide data day and night, regardless of cloud cover. Several past studies demonstrated that SAR systems are suitable tools for flood mapping so that the use of SAR data is presently well-established in operational services for flood management. For the purpose of a timely and effective flood management, a number of algorithms published in the literature are available to produce near real time (NRT) flood maps (Chini et al., 2013; Pulvirenti et al., 2011; Chini et al., Under Review). In spite of this progress in the development of NRT flood mapping procedures, the detection of inundation in vegetated and urban areas still represents a critical issue (Chini et al., 2012; Pulvirenti et al., 2013; Pulvirenti et al., 2016). This is due to the fact that radar signatures of such targets are often ambiguous. As a matter of fact, there is clearly a necessity to make use of the enhanced observational capabilities of Sentinel-1 in order to find better ways for detecting floodwater in vegetated and urban areas. Indeed the dual-pol acquisitions mode, the medium spatial resolution $(20 \mathrm{~m})$ with a huge swath width $(250$ $\mathrm{Km}$ ), the high repeat cycle (small temporal baseline) and relative narrow orbit tube (small perpendicular baseline in case of interferometric acquisitions) are all characteristics that make momentum for the development of new automatic flood mapping algorithms in more complex land cover environment.

In the present work, the high sensitivity of the interferometric coherence to detect small changes has been exploited to detect the presence of water in urban area, but also to reduce possible false alarms caused by soil moisture effects in case of base soil and also to extract information about land cover, replacing in this respect optical data features such as Normalized Difference Vegetation Index (NDVI). Instead, the polarimetric characteristics of Sentinel-1 have been used to detect flood in vegetated area, since the cross- and co-polarized backscattering behave differently respect to the double-bounce between vertical structures and the water on surface. All these effects have been taken into account making use of a change detection approach where changes of the backscattering at the different polarizations, co- and cross-, and the interferometric coherence respect their dry-state have driven the detection of flooded pixels in a statistical based framework. The test case is the Evros River, the second largest river in Eastern Europe after Danube River, flowing through Bulgaria, Greece and Turkey discharging into the north Aegean Sea.

\section{Methods}

The approach tries to identify the flood that occurs in three main land cover classes, such as urban areas, bare or poorly vegetated soil and vegetated areas, taking advantage of co- and cross-polarized SAR backscattering channels, and the InSAR coherence to better characterize the landscape.

In a SAR image, the amplitude and phase of the backscatter field depends on the physical (i.e., geometry, roughness) and electrical (i.e., dielectric constant) properties of the target. The presence of floodwater changes these properties because the ground roughness tends to become smoother and the real part of the dielectric constant becomes considerably larger than that of both dry and moist soils. As a result, the surface reflectivity increases.

In bare soil the decreased roughness let the backscattering decrease and this behaviour is the same in both co- and cross-polarized channel, thus we identify the insurgence of flood taking into account just the backscattering intensity. 
In urban area, the double bounce effect is typically represented in SAR images by a very bright line that appears on the side of the wall that is illuminated by the radar (Franceschetti et al., 2002). It is worth mentioning that double bounce paths all have the same length as the range of the intersection between the ground and the front wall of the building. In this case, even if the building is surrounded by smooth asphalt surfaces, so that the change in soil roughness may be negligible, the increase of reflectivity due to the increase of the dielectric constant implies, in principle, a considerable increase of the double bounce effect. If we consider the angle between the flight direction and the street alignment, the increase is high for small angles while is reduced for higher angles. As a consequence, even in an ideal situation of an isolated building surrounded by a homogenous ground surface, the increase of the double bounce due to the presence of floodwater is not very high if buildings are not parallel to the SAR flight direction. This increase can be hardly detected using only the backscatter intensity in a complex urban environment. Hence, the idea is to integrate intensity data with the InSAR coherence, which is basically a measurement of the degree of correlation between two complex SAR images. It is defined as the normalized cross correlation between the images and it is related to the change in the spatial arrangement of the scatterers within a SAR image pixel (Chini $e t$ al., 2015), and thus to geometric changes in the scene. An interferometric pair can be built using two images taken before and after the flood, and it is expected that flooded areas exhibit low coherence, typical feature to distinguish the presence of water, especially in urban areas where this feature is usually very high regardless the images temporal baseline.

Concerning vegetated areas, which are more complex environment, are usually characterized by low coherence due to changes in plant growing stage. Subsequently, coherence can help distinguishing increases of backscatter due to soil moisture variations, in case of bare soil, from those due to the presence of water beneath vegetation, double-bounce phenomenon, where coherence is considerably lower. In this case the coherence plays basically the role of replacing optical data to detect vegetation. Furthermore, we can take advantage of the two polarization available. Indeed the cross-polarized backscatter is more sensible to volume scattering, while is the co-polarized one the more sensible to the double bounce. As a matter of fact, in vegetated areas we should detect a sensible backscatter increase in the VV polarization and not in the VH one (Hess et al., 1990).

\section{Results}

As already mentioned in the introduction, the test case is the Evros River, which flows through Bulgaria, Greece and Turkey discharging into the north Aegean Sea. The river has a total length of about $515 \mathrm{~km}$ of which 218 flowing along the Greece - Turkey border and the rest in Bulgaria. The Evros river catchment, of $52,900 \mathrm{~km}^{2}$ is one of the most intensively cultivated areas in the Balkans with a high socioeconomic and environmental (Evros Delta is protected under Ramsar Convention) importance. Although the last fifty years significant hydraulic works have been constructed several severe floods events struck the catchment and particularly the southern part the most severe of them happen during 2004, 2005, 2006, 2009 and during the current year causing severe damages to agriculture, transport and water supply networks. In particular, in the period between October 2014 and August 2015 have been collected more than twenty dual-pol Sentinel-1 images (VV/VH) making possible to monitor the flood evolution. The flood is present in almost all images, except two, which have been taken as reference, the images acquired on October 13 and 25, 2014.

The available SAR data have been calibrated and geocoded by means of the ESA-SNAP Toolbox software to derive the backscattering coefficient $\left(\sigma_{0}\right)$. For this purpose, the SRTM 3 sec DEM has been used (Farr et al.). The products have been multilooked, 5 looks in range and 1 in azimuth, as well as a pixel size of $20 \times 20 \mathrm{~m}^{2}$ has been chosen for the Sentinel-1A calibrated data. As for the coherence, it has also been extracted through the ESA-SNAP Toolbox software considering a moving window size of $7 \times 7$ pixels. All products have been co-registered as a first step of the data processing. 
In Figure 1 is shown an example of the detection of flood in bare soil and vegetated areas, such as marshes. In particular it is highlighted the advantage of using dual-pol data (VV/VH). It is worth to note how in base soil areas the decrease of the backscatter occurs for both polarizations (red areas) while for the vegetated ones the increase is evident only in VV (light blue areas).

In Figure 2 is shown an example of the role of the coherence for detecting flood in urban and vegetated areas. There are highlighted two different RGB colour composition representations:

- Left: coherence between images pre-flood (October 25, 2014) and post-flood (November 6, 2014) $(\mathrm{R})$, intensity VV pre-flood $(\mathrm{G})$ and intensity VV post-flood (B). White areas are representative of unchanged areas, while green and blue regions are where the flood occurred in bare soil and vegetated areas respectively.

- Right: coherence pre-flood (October 13, 2014-October 25, 2014) (R), and coherence post-flood (October 25, 2014-November 6, 2014) (G and B). Red areas represent where the coherence is high in the pre-event coherence map while the light blue ones those ones where the coherence is higher in post-flood one.

Moreover, in Figure 2 the examples of bare soil (upper panel), vegetated areas (central panel) and urban areas (lower panel) are shown.

In the bare soil class the flood is depicted in green (left, low coherence and higher backscattering in the pre-event image), the pre-event coherence is higher as well (right).

In the vegetated areas the flood is highlighted in blue (left, low coherence and higher backscattering in the post-event image), while in this case the pre-event coherence is low as well (right, dark areas).

Finally, urban areas, that fortunately were not affected by flood, are quite bright (left, high coherence and high intensity in both images), and as well high coherences in the pre- and post-event maps (right). 

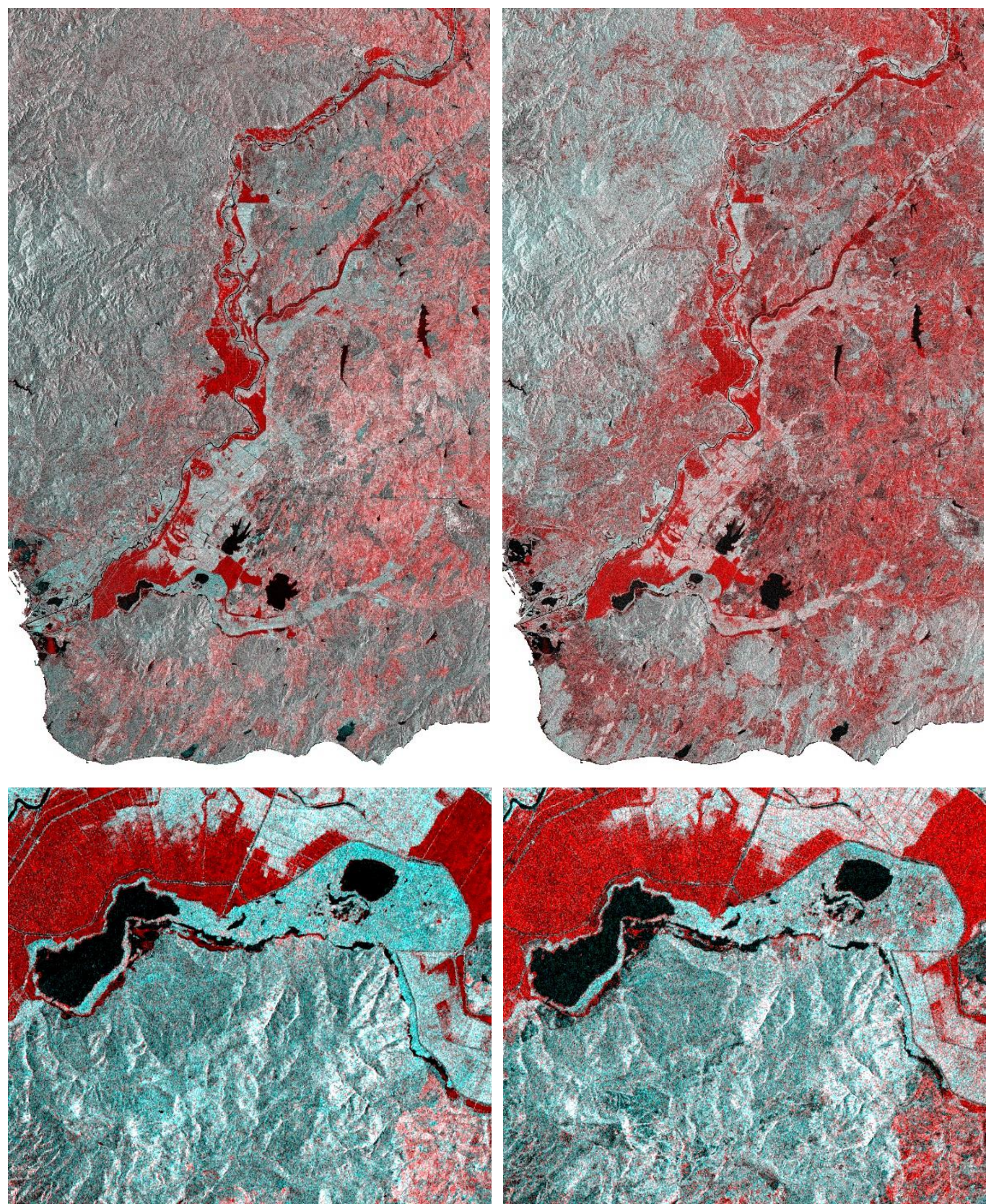

Figure 1 - RGB combination involving the October 25, 2014 intensity image (R), the March 3, 2015 intensity image (G and B), VV (left) and VH (right polarization. The red areas in the image represent bare soil which are flooded in both polarizations in the second date. Light blue areas represent the flooded vegetation, the increase of backscatter is quite evident in the VV polarization (left). In the lower panel a detail of the upper one is shown in both polarizations, VV (left) and VH (right). 


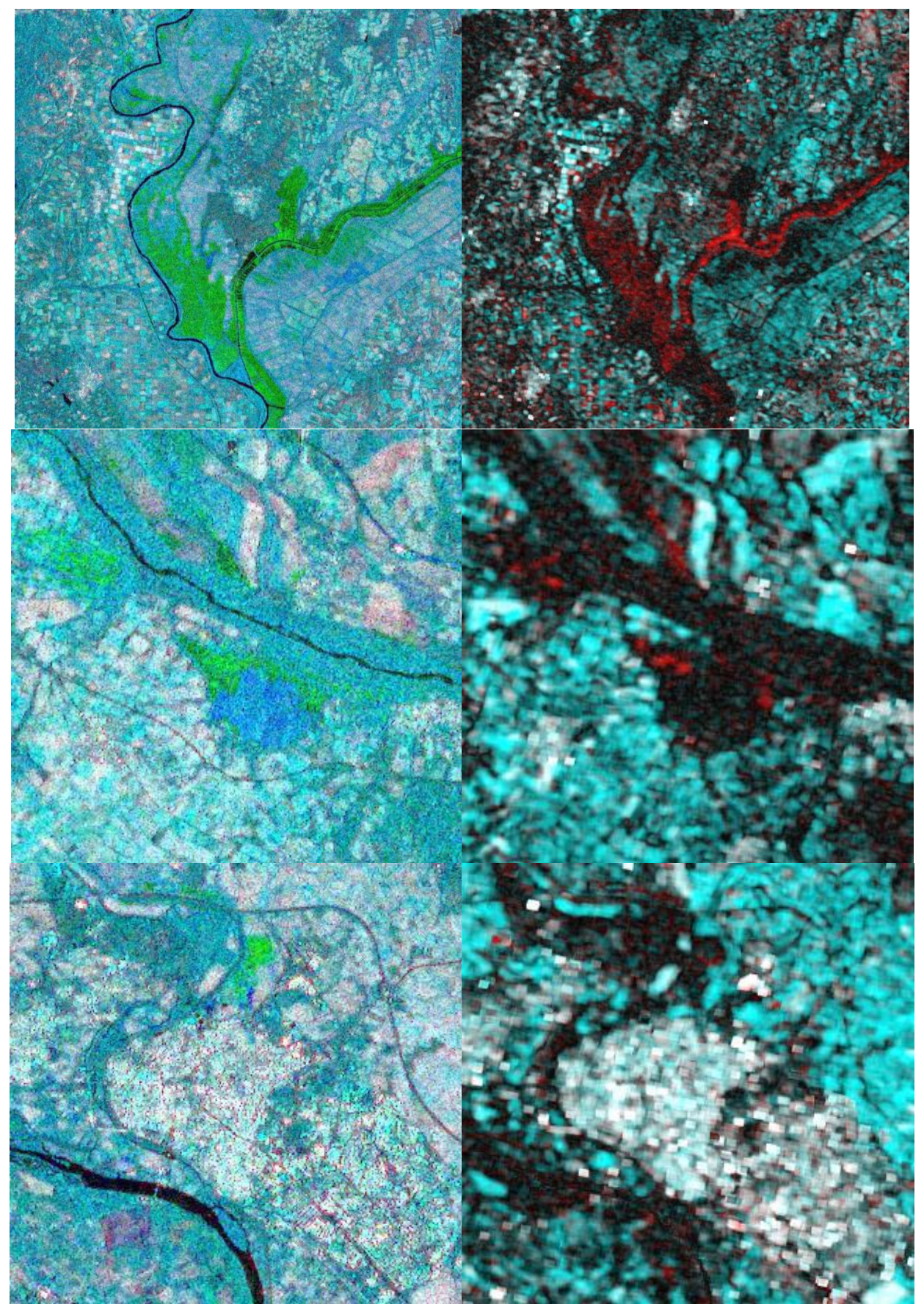

Figure 2 - RGB colour composition. Left: Coherence between image pre-flood (October 25, 2014) and post-flood (November 6, 2014) (R), intensity VV pre-flood (G) and intensity VV post-flood (B). Green and blue regions are where the flood occurred in bare soil and vegetated areas respectively. Right: Coherence pre-flood (October 13, 2014-October 25, 2014) (R), and coherence post-flood (October 25, 2014-November 6, 2014) (G and B). Red areas represent where the coherence is high in the pre-event coherence map while the light blue areas those ones where the coherence is higher in post-flood one. 


\section{Acknowledgments}

Marco Chini's contribution was supported by the National Research Fund of Luxembourg through the PAPARAZZI project (COREC11/SR/1277979).

Part of the research was carried out during the stay of Asterios Papastergios in the Luxembourg Institute of Science and Technology under the frame of the Erasmus placement program, under the supervision of Professor Issaak Parcharidis and Dr Marco Chini.

\section{References}

Chini, M., Pulvirenti, L. and Pierdicca, N., 2012. Analysis and interpretation of the COSMOSkyMed observations of the 2011 Japan Tsunami, IEEE Geosci. Remote Sens. Lett., 9(3), 467-471.

Chini, M., Piscini, A., Cinti, F.R., Amici, S., Nappi, R. and De Martini, P.M., 2013. The 2011 Tohoku-Oki (Japan) tsunami inundation and liquefaction investigated by optical, thermal and SAR data, IEEE Geosciences and Remote Sensing Letters, 10(2), 347-351.

Chini, M., Albano, M., Saroli, M., Pulvirenti, L., Moro, M., Bignami, C., Falcucci, E., Gori, S., Modoni, G., Pierdicca, N. and Stramondo, S., 2015. Co-seismic liquefaction phenomenon analysis by COSMO-SkyMed: 2012 Emilia (Italy) earthquake, International Journal of Applied Earth Observation and Geoinformation, 39, 65-78.

Chini, M., Hostache, R., Giustarini, L. and Matgen, P., Under Review. A Hierarchical Split-Based Approach (HSBA) for automatically mapping flooded areas using SAR images of variable size and resolution, IEEE Transactions on Geoscience and Remote Sensing.

Franceschetti, G., Iodice, A. and Riccio, D., 2002. A canonical problem in electromagnetic backscattering from buildings, IEEE Trans. Geosci. Remote Sens., 40(8), 1787-1801.

Hess, L.L., Melack, J.M. and Simonett, D.S., 1990. Radar detection of flooding beneath the forest canopy: A review, Int. J. Remote Sens., 11, 1313-1325.

Pulvirenti, L., Pierdicca, N., Chini, M. and Guerriero, L., 2011. An algorithm for operational flood mapping from synthetic aperture radar (SAR) data based on the fuzzy logic, Nat. Hazards Earth Syst. Sci., 11, 529-540.

Pulvirenti, L., Pierdicca, N., Chini, M. and Guerriero, L., 2013. Monitoring flood evolution in vegetated areas using COSMO-SkyMed data: The Tuscany2009 case study, IEEE J. Sel. Topics Appl. Earth Observ. Remote Sens., 6(4), 1807-1816.

Pulvirenti, L.,Chini, M., Pierdicca, N. and Boni, G., 2016. Use of SAR data for detecting floodwater in urban and suburban areas: the role of the interferometric coherence, IEEE Transactions on Geoscience and Remote Sensing, doi: 10.1109/TGRS.2015.2482001. 